\title{
ARTICLE OPEN Coherent control and high-fidelity readout of chromium ions in commercial silicon carbide
}

\author{
Berk Diler (D) ${ }^{1}$, Samuel J. Whiteley $\mathbb{D}^{1,2}$, Christopher P. Anderson (iD ${ }^{1,2}$, Gary Wolfowicz $\mathbb{D}^{1,3}$, Marie E. Wesson ${ }^{2}$, Edward S. Bielejec ${ }^{4}$ \\ F. Joseph Heremans (iD ${ }^{1,3}$ and David D. Awschalom (iD) ${ }^{1,2,3 *}$
}

Transition metal ions provide a rich set of optically active defect spins in wide bandgap semiconductors. Chromium (Cr $\left.{ }^{4+}\right)$ in siliconcarbide $(\mathrm{SiC})$ produces a spin-1 ground state with a narrow, spectrally isolated, spin-selective, near-telecom optical interface. However, previous studies were hindered by material quality resulting in limited coherent control. In this work, we implant $\mathrm{Cr}$ into commercial $4 \mathrm{H}-\mathrm{SiC}$ and show optimal defect activation after annealing above $1600{ }^{\circ} \mathrm{C}$. We measure an ensemble optical hole linewidth of $31 \mathrm{MHz}$, an order of magnitude improvement compared to as-grown samples. An in-depth exploration of optical and spin dynamics reveals efficient spin polarization, coherent control, and readout with high fidelity (79\%). We report $T_{1}$ times greater than $1 \mathrm{~s}$ at cryogenic temperatures $(15 \mathrm{~K})$ with a $T_{2}{ }^{*}=317 \mathrm{~ns}$ and a $T_{2}=81 \mu \mathrm{s}$, where spin dephasing times are currently limited by spin-spin interactions within the defect ensemble. Our results demonstrate the potential of $\mathrm{Cr}^{4+}$ in $\mathrm{SiC}$ as an extrinsic, optically active spin qubit.

npj Quantum Information (2020)6:11; https://doi.org/10.1038/s41534-020-0247-7

\section{INTRODUCTION}

Optically active defect spins in wide bandgap semiconductors have attracted much attention as candidate qubits for quantum information. ${ }^{1,2}$ These defects hold promise due to their long lived, highly localized ground state spin states. They can be manipulated using a number of quantum control mechanisms ${ }^{3}$ and interfaced with optics through atom-like spin-selective transitions for longdistance quantum communication and entanglement. ${ }^{4}$ The wide range of available point defects, including complexes and vacancies in diamond ${ }^{4-6}$ and silicon-carbide $(\mathrm{SiC})^{7-10}$ as well as rare-earth ions in oxides, ${ }^{11}$ offer many possible electronic, spin, and optical properties depending on their host materials. As each host provides different benefits, it is desirable to decouple the defect properties from their hosts for reliable defect qubit integration into semiconducting devices.

The prototypical host-independent optically active defects are rare-earth ions, since the transitions within their partially filled $f$ orbitals are shielded by their outer electronic shells. ${ }^{12}$ However, their magnetic fine structure within the orbitals due to the crystal field is highly host dependent and is difficult to predict. ${ }^{13}$ Furthermore, the weak dipole moment of these orbitals result in millisecond long excited state lifetimes which makes optical spin polarization and detecting a single emitter challenging without integrated nanophotonics. ${ }^{14-16}$

Transition metal ions have shown to be host-independent by preserving the physics of the defect center across multiple platforms. $^{10}$ This is enabled by the similar defect $d$-orbital electronic structures between various hosts that share lattice symmetry and have similar bond strength. ${ }^{17}$ Transition metal systems in $\mathrm{SiC}$ have already attracted attention as optically active spin qubits such as $\mathrm{V}^{4+} 18,19$ and $\mathrm{Mo}^{5+20}$ Both defects possess only one electron in their $d$-orbitals, resulting in a $3 d^{1}$ and $4 d^{1}$ configuration, respectively, with a spin- $-1 / 2$ ground and excited states. Under the SiC ligand symmetry the ground state is expected to be comprised of multiple close orbitals which could result in short spin-lattice relaxation $\left(T_{1}\right)$ times. ${ }^{19,21}$

By contrast, chromium defects in $\mathrm{SiC}$ in the $4+$ charge state $\left(\mathrm{Cr}^{4+}\right)$ are in the $3 d^{2}$ electronic configuration, forming a ${ }^{3} \mathrm{~A}_{2}$, orbital singlet spin triplet ground state and a ${ }^{1} \mathrm{E}$ spin singlet orbital doublet excited state. The non-degenerate nature of the ground state orbital yields long spin $T_{1}$ times while the low nuclear spin density of the $\mathrm{SiC}$ host should enable long spin coherence times $\left(T_{2}\right)$. Even though the optical transition between ${ }^{1} E$ and ${ }^{3} A_{2}$ states is spin forbidden, resulting in a long decay time $(146 \mu \mathrm{s})$, both states are formed by the different electron configuration of the same orbitals, creating a weak Jahn-Teller coupling ${ }^{22}$ which leads to a very high Debye-Waller factor of at least $75 \% .^{10}$ This wellisolated transition lies in the near-telecom range $(1070 \mathrm{~nm})$, making $\mathrm{Cr}^{4+}$ particularly interesting for in-fiber long distance quantum communication with integrated spins. However, the previous transition metal ion studies in $\mathrm{SiC}$ including $\mathrm{Cr}^{4+}$ have been mostly conducted on as-grown samples ${ }^{10,18,20}$ and their coherent ground state spin characterization is yet to be demonstrated.

In this work, we study the creation of $\mathrm{Cr}^{4+}$ defect spins in commercial SiC through implantation and annealing, a critical step towards accurate three-dimensional localization of spin defects for device integration. ${ }^{23}$ Since the optical transition is rather insensitive to environmental perturbations like strain, the fine structure of an ensemble of $\mathrm{Cr}^{4+}$ can be resolved under resonant excitation. This property combined with the $\Lambda$-like configuration of the spin forbidden optical transitions makes ensemble spin polarization and readout possible. We therefore demonstrate high fidelity coherent control of the $\mathrm{Cr}^{4+}$ ensemble by Rabi oscillations with contrast exceeding $79 \%$. We then characterize the ground state spin coherence times of $\mathrm{Cr}^{4+}$ in $\mathrm{SiC}$ and find long longitudinal spin relaxation times $\left(T_{1}\right)$ at cryogenic temperatures and spin-dephasing relaxation time $\left(T_{2}\right)$ over $80 \mu \mathrm{s}$, similar to other spin defects in $\mathrm{SiC}$ with comparable ensemble densities. ${ }^{24}$ The 
transportable nature of the electronic configuration and the lattice insensitive narrow optical transitions combined with long spin coherence times that can be read out with high fidelity, makes $\mathrm{Cr}^{4+}$ in $\mathrm{SiC}$ and more generally, the $d^{2}$ configuration in strong tetrahedral ligand field, a promising optically active, transportable and localizable spin qubit.

\section{RESULTS}

Creation and activation of $\mathrm{Cr}^{4+}$ in commercial $\mathrm{SiC}$ is a critical step for devices with integrated, optically accessible spins. To this end we introduce isotopically pure, nuclear spin-free ${ }^{52} \mathrm{Cr}$ atoms into commercially purchased $4 \mathrm{H}-\mathrm{SiC}$ substrates through implantation at elevated temperatures up to $700^{\circ} \mathrm{C}$. We believe that the implanted chromium atoms sit mainly in interstitial sites of the $\mathrm{SiC}$ lattice. During the subsequent annealing, $\mathrm{Cr}$ atoms move to the silicon ( $\mathrm{Si}$ ) site and form bonds with the surrounding carbon atoms (Fig. 1a). This generates the $\mathrm{Cr}^{4+}$ level structure shown in Fig. 1b (defect activation). The two different Si lattice sites in $4 \mathrm{H}-$ $\mathrm{SiC}$ result in two distinct $\mathrm{Cr}^{4+}$ species: $\mathrm{Cr}_{\mathrm{A}}$ (quasi-cubic k-site) and $\mathrm{Cr}_{C}$ (quasi-hexagonal h-site). ${ }^{22}$ To investigate the electrical activation of the defect ensembles, we anneal different samples from the same implanted substrate between 800 and $1800^{\circ} \mathrm{C}$ and then measure the zero-phonon line (ZPL) intensities of $\mathrm{Cr}_{A}$ and $\mathrm{Cr}_{C}$ (Fig. 1c). $\mathrm{Cr}^{4+}$ activation, not observable when the samples are not annealed, increases monotonically as a function of annealing temperature until $1600^{\circ} \mathrm{C}$ where it saturates.

For coherent characterization of the defect, we create $\mathrm{Cr}^{4+}$ in a wider range of depth in $\mathrm{SiC}$ by combining multiple high energy ion implantations (see Methods) followed by $1800^{\circ} \mathrm{C}$ annealing to reduce the overall $\mathrm{Cr}^{4+}$ density while maximizing its signal. Offresonant photoluminescence $(\mathrm{PL})$ at $30 \mathrm{~K}$ demonstrates successful activation of $\mathrm{Cr}^{4+}$ in this sample (Fig. 1d). The weak spin-forbidden transition of $\mathrm{Cr}^{4+}$ under resonant excitation is challenging to detect in the presence of other four orders of magnitude brighter optically active residual defects (such as the divacancy ${ }^{8}$ ) created during substrate growth and ion implantation. However, the longlived excited state of $\mathrm{Cr}^{4+}$ enables temporal separation of the relevant $\mathrm{PL}$ from the background (see $\mathrm{SI}$ ) by only integrating over the transient signal. We measure the photoluminescence excitation (PLE) spectrum by resonantly exciting $\mathrm{Cr}^{4+} \mathrm{ZPL}$ and collecting the transient phonon sideband (Fig. 1e) at $30 \mathrm{~K}$. A fit to two Gaussian functions for the $m_{\mathrm{s}}=0$ and $m_{\mathrm{s}}= \pm 1$ sublevels with a known ground state crystal field splitting $(D)$ for $\mathrm{Cr}_{\mathrm{A}}(1063 \mathrm{MHz})$ yields an average PLE linewidth of $5.1(4) \mathrm{GHz}$ with the $m_{\mathrm{s}}=0$ linewidth roughly two times larger than the $m_{s}= \pm 1$ linewidth. The origin of this behavior is unknown and may be a result of mass related shift due to ${ }^{13} \mathrm{C},{ }^{29} \mathrm{Si}$, and ${ }^{30} \mathrm{Si}$ isotopes. ${ }^{19,25,26} \mathrm{We}$ observe a similar trend for $\mathrm{Cr}_{\mathrm{C}}$ (see $\mathrm{SI}$ ) where the individual PLE lines are more resolved due to a larger $D$. The overall sharper inhomogeneous linewidth of the implanted sample compared to the as-grown sample ${ }^{10}$ allows for better spin sublevel resolution and is likely due to an improved spatial strain profile.

We then characterize the ground state spins of $\mathrm{Cr}^{4+}$ in $\mathrm{SiC}$ through resonant hole burning and hole recovery at $15 \mathrm{~K}$ where the spin $T_{1}$ is longer than the optical lifetime. The population of a spin sub-ensemble excited by the narrow line resonant laser will be redistributed among the other ground state spin sublevels after spontaneous emission, leading to hole burning and spin polarization. Since the polarized sub-population is off-resonant relative to the probe laser, the defect goes dark resulting in a spin selective optical contrast. We can recover the hole by reintroducing the polarized population into the optical cycle in two ways, either by using a second laser color to probe the polarized sublevels or by driving ground state spins with microwaves. A second laser tone generated by a phase modulator recovers the $\mathrm{Cr}_{\mathrm{A}}$ hole at a $D$ of 1062.7(4) $\mathrm{MHz}$ (Fig. 2a). A Lorentzian fit to the data extracts a $31(2) \mathrm{MHz}$ ensemble hole linewidth, which is more

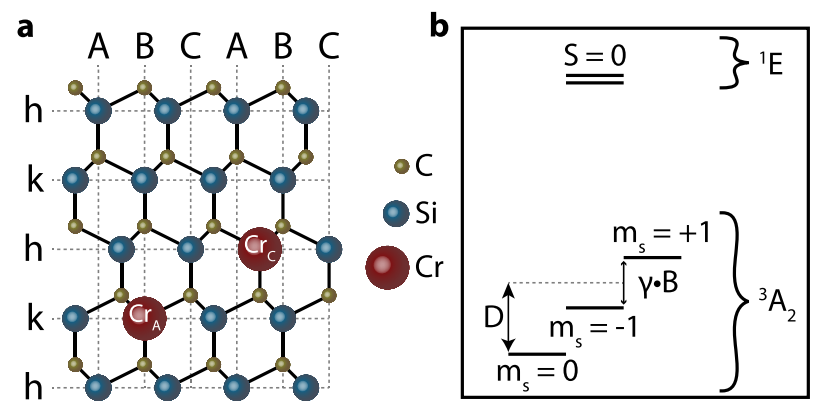

C
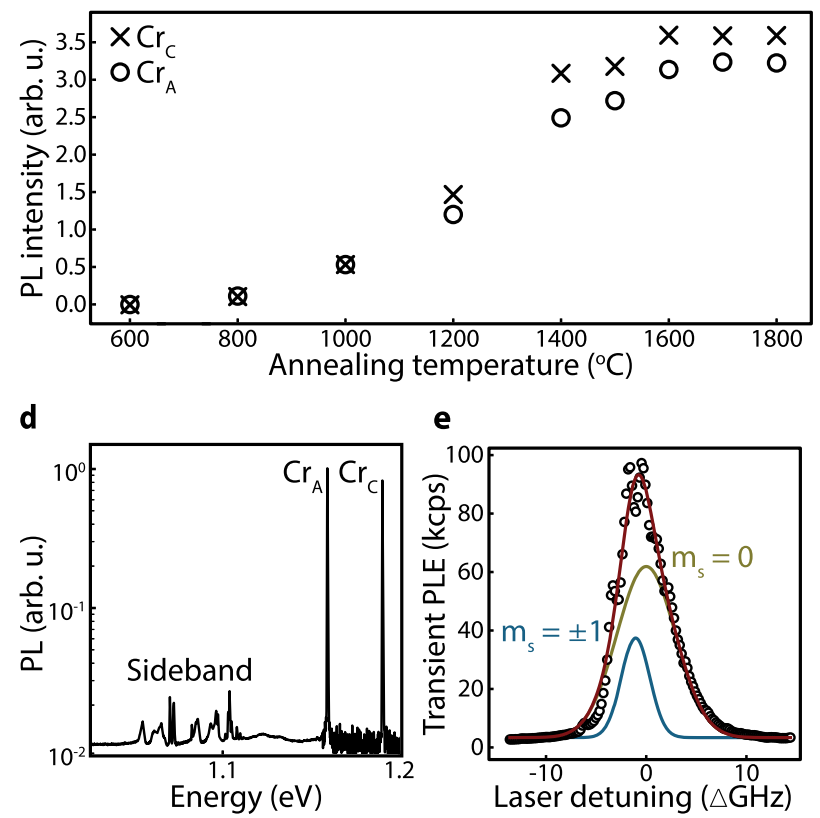

Fig. 1 Structure, creation and spectroscopy of chromium defects in $4 \mathrm{H}-\mathrm{SiC}$. a An illustration of substitutional $\mathrm{Cr}^{4+}$ ions in silicon sites of a $4 \mathrm{H}-\mathrm{SiC}$ lattice adapted from ref. ${ }^{34}$ b The electronic level structure of $\mathrm{Cr}^{4+}$ in SiC. The $m_{\mathrm{s}}=0$ and $m_{\mathrm{s}}= \pm 1$ sublevels are split by the crystal field $(D)$, and under a magnetic field, the $m_{\mathrm{s}}= \pm$ 1 sublevels are Zeeman split. c Cr ${ }^{4+}$ activation as a function of annealing temperature is measured by integrating the zero-phonon lines (ZPLs) intensity of the photoluminescence under off-resonant $(730 \mathrm{~nm})$ excitation at $T=30 \mathrm{~K}$. d A photoluminescence spectrum of the sample used for spin and optical control at $T=30 \mathrm{~K} . \mathrm{Cr}_{\mathrm{A}}$ and $\mathrm{Cr}_{\mathrm{C}}$, two different sites of $\mathrm{Cr}^{4+}$ in $4 \mathrm{H}-\mathrm{SiC}$, ZPL's and their sidebands can be observed. e $\mathrm{Cr}_{\mathrm{A}}$ photoluminescence excitation (PLE) at $T=30 \mathrm{~K}$ is measured by sweeping the resonant laser and recording the transient sideband signal in counts per second (cps). The PLE is fit to two Gaussian peaks with a known $D=1063 \mathrm{MHz}$ splitting. ${ }^{10}$ The full width at half maxima are $6.87(27) \mathrm{GHz}$ for $m_{\mathrm{s}}=0$ and $3.34(39)$ $\mathrm{GHz}$ for $m_{\mathrm{s}}= \pm 1$ peak. The one sigma errors of the data are smaller than the point size and are not displayed.

than an order of magnitude narrower than the as grown samples, ${ }^{10}$ demonstrating the superior material quality of the implanted and annealed sample. However, the sharp hole linewidth is also two orders of magnitude narrower than the inhomogeneous optical linewidth, indicating only $1 \%$ of the created $\mathrm{Cr}_{\mathrm{A}}$ defects are accessed under resonant excitation. One could increase the net ensemble polarization by further reducing the inhomogeneous linewidth through material improvements. The hole can also be recovered by microwave mixing of the ground state spin sublevels, enabling optical detection of magnetic resonance (ODMR) (Fig. 2b). Stray magnetic fields Zeeman split the $m_{\mathrm{s}}= \pm 1$ ODMR by $\sim 5 \mathrm{MHz}$ centered around $D$ 
$=1063.11$ (1) $\mathrm{MHz}$ (Fig. 2b). A Lorentzian fit to the non-power broadened ODMR reveals a linewidth of 1.32(2) MHz. Because the $31 \mathrm{MHz}$ optical hole linewidth is not confined by the ODMR linewidth, the optical coherence is likely excited-state limited. The four orders of magnitude difference between the measured and lifetime limited hole linewidth $(\sim 2 \mathrm{kHz})$ may be a result of decoherence induced by the degenerate excited state orbital doublet structure that can be lifted by applying high magnetic fields ${ }^{22}$ or spectral diffusion due to charge fluctuations that can be addressed by creating charge depletion zones; ${ }^{27}$ the exact mechanism and its solution would require further investigation.

A c-axis magnetic field lifts the optical degeneracy of the $m_{\mathrm{s}}=$ \pm 1 sublevels by Zeeman splitting while the $S=0$ excited state remains unaffected (Fig. $1 \mathrm{~b}$ ). It is worth noting that the population can become trapped in the unprobed third level after using either the optical sideband probe or the microwave mixing, as these techniques can only address one sub-level at a time. By applying both an optical sideband probe and a microwave tone together, all states can be addressed simultaneously resulting in hole recovery (Fig. 2c). Using a second laser to trap the population into the third sublevel provides a path for efficient spin polarization under a magnetic field.

For coherent control and readout of $\mathrm{Cr}^{4+}$, we polarize a subpopulation into the $m_{\mathrm{s}}=+1$ sublevel by tuning the primary laser color to be resonant with $m_{\mathrm{s}}=0$ state and adding a sideband tone to optically excite the $m_{\mathrm{s}}=-1$ sublevel (see $\mathrm{SI}$ ). Once

a

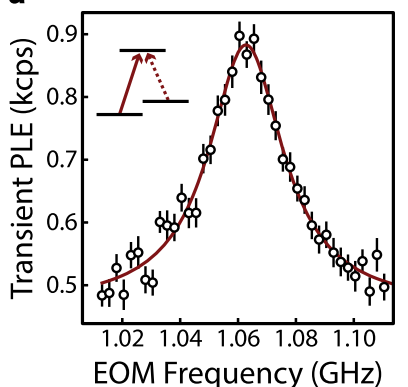

C

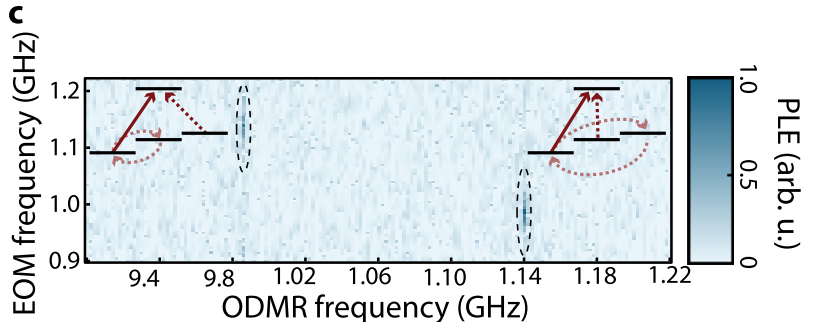

polarized, we coherently control the subpopulation using resonant microwave rotations within the $m_{\mathrm{s}}=0,+1$ manifold and read out the $m_{\mathrm{s}}=0$ population with a short ( $\left.50 \mu \mathrm{s}\right)$ primary laser excitation (Fig. 3a). We measure Rabi rotations at $B=158 \mathrm{G}$ by varying the microwave excitation length and obtain a contrast of $63(1) \%$ with an envelope decay time of $4.76(7) \mu$ s (Fig. 3b).

To characterize the ground state spin coherence times, we perform Ramsey interferometry and Hahn echo measurements. For the Ramsey measurement, we detune the resonant microwave frequency by $5 \mathrm{MHz}$ and delay the time between two $\pi_{x} / 2$ pulses. The characteristic oscillations at the detuning frequency have an envelope with a decay time of $T_{2}^{*}=307(17)$ ns (Fig. 3c), in agreement with the ODMR linewidth measurements in Fig. 2b. We measure the $T_{2}$ coherence time by $\left( \pm \pi_{\mathrm{x}} / 2-t_{\text {free }} / 2-\pi_{\mathrm{y}}-t_{\text {free }} / 2-\right.$ $\pi_{\mathrm{x}} / 2$ ) echo-pulse sequence. We fit the data to an electron spin echo envelope modulation function where nuclear Larmor precessions are enveloped by a decay function of $e^{-\left(t / T_{2}\right)^{n}} \Pi\left(1-K_{\mathrm{a}} \sin ^{2}\left(\pi \omega_{a} \tau\right)\right)$, with the precession amplitudes $\left(K_{\mathrm{a}}\right)$, frequencies $\left(\omega_{\mathrm{a}}\right)$ and decay power $(n)$ and time $\left(T_{2}\right)$ as free fit parameters. ${ }^{28}$ We recover the characteristic oscillations at the current $B$ field for ${ }^{13} \mathrm{C}(87.5(3) \mathrm{kHz})$ and ${ }^{29} \mathrm{Si}(68.0(1) \mathrm{kHz})$ as well as $T_{2}=81(2) \mu s$ with a decay power dependence of $n=1.9(1)$ (Fig. $3 d)$. The average defect density in our sample is roughly $3 \times 10^{16}$ atoms $/ \mathrm{cm}^{3}$ (see SI) and the measured $T_{2}$ time is in line with similarly dense divacancy ensembles in $\mathrm{SiC}^{24}$ This indicates that the observed coherence times are likely limited by electron spin-spin interactions between defects, and further reduction of the $\mathrm{Cr}^{4+}$ and background defect densities could result in millisecond long coherence times. ${ }^{28}$

Finally, we report that by sacrificing overall signal and reducing the probe time from 50 to $1 \mu \mathrm{s}$ we increase the Rabi contrast to 79 (2)\% contrast (Fig. 3f). This places a lower bound on the ensemble polarization of at least $77 \%$. The contrast, however, is lower than our estimate (see $\mathrm{SI}$ ) and we believe a spin-reset mechanism induced by the probe itself could be responsible, therefore, further exploration of this effect could increase the readout fidelity.

The spin characterization measurements reported above are enabled by the careful study of the rates responsible for the $\mathrm{Cr}^{4+}$ population dynamics. One such rate is the excited state lifetime $\left(T_{\text {opt }}\right)$ under resonant excitation which we measure at $30 \mathrm{~K}$ by histogramming the transient PLE to find $T_{\text {opt }}=156.3(5) \mu$ s (Fig. 4a). This is in agreement with the off-resonant excited state lifetime $^{10}$ (see SI). Based on this measurement, our integration window for the collected photons in the transient readout is $155 \mu$ s long.

To understand the ground state spin dynamics, we measure the spin $T_{1}$ time at $B=0 \mathrm{G}$ by population inversion relaxation using a microwave $\pi$-pulse. By repeating this measurement for temperatures between 15 and $30 \mathrm{~K}$ and fitting each data set to an exponential decay function, we extract $T_{1}$ times and use this dataset to distinguish between Orbach and Raman processes ${ }^{29}$ (Fig. 4b). For the Orbach process, we use $\frac{1}{T_{1}}=A e^{-\frac{E}{k_{B} T}}$ as our fit function where $A$ and $E$, the energy difference to a low-lying excited state, are fit parameters. For the Raman process we use $\frac{1}{T_{1}}=A(T-\Delta T)^{n}$ where $A$ and $\Delta T$, a constant temperature offset of the sample, are fit parameters for various fixed odd integers $n$. The Orbach model fits the data poorly and returns an energy gap of $E=20$ (1) meV which is a factor of 50 lower from the first excited state energy and a factor of 4 lower than the closest phonon line energy, ${ }^{22}$ indicating that Orbach process is not the likely explanation for $T_{1}$ decay mechanism. Out of the Raman processes, the most likely explanation is the $n=9$ process (see SI), indicating that the $T_{1}$ is spin-orbit interaction limited. The longest measured spin $T_{1}$ time is $1.6(3) \mathrm{s}$ long at $T=15 \mathrm{~K}$, long enough to not limit the spin dephasing times and to offer a very long window for high fidelity spin initialization. 
a

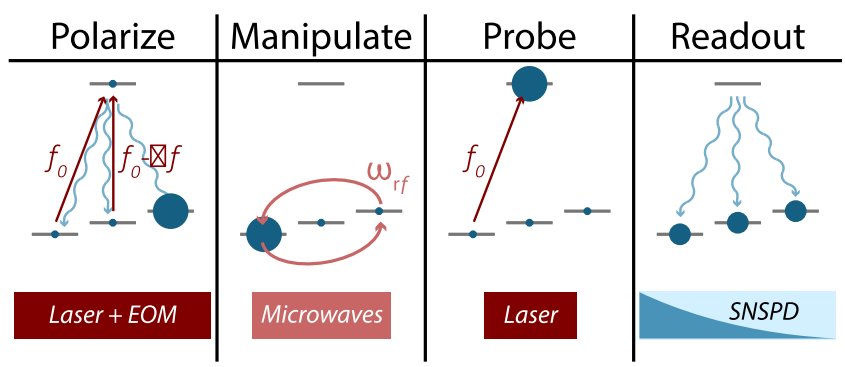

C

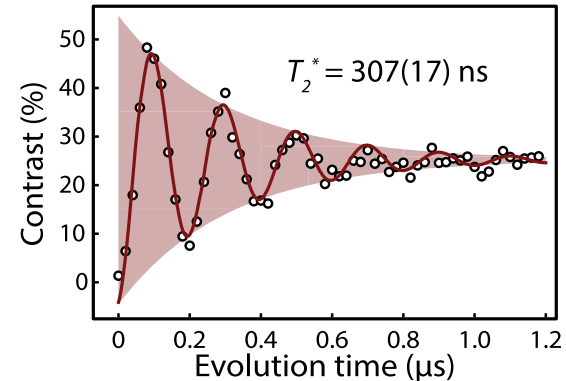

d

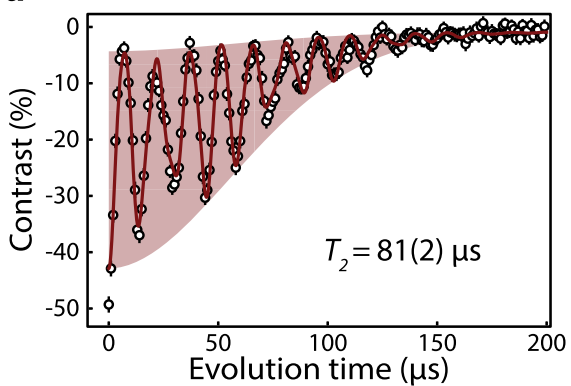

b

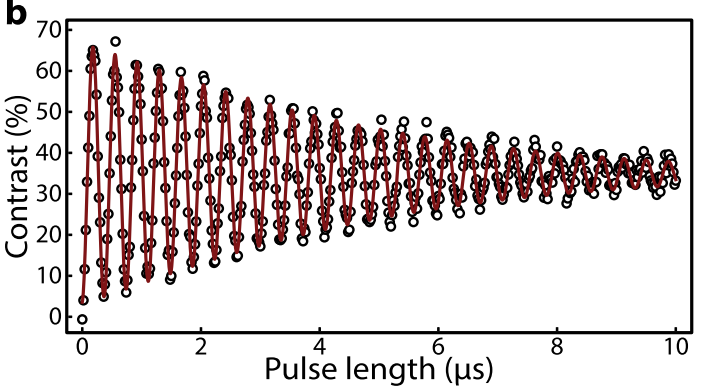

e

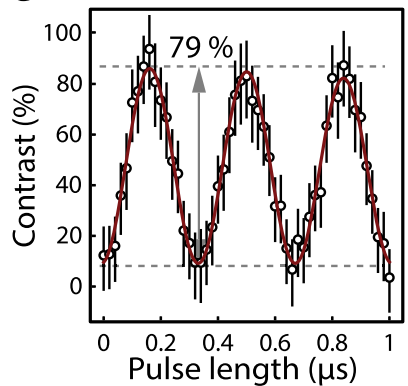

Fig. 3 Coherent control of $\mathbf{4 H}-\mathrm{SiC}_{\mathbf{C}} \mathrm{Cr}_{\mathrm{A}}$ at $\boldsymbol{T}=\mathbf{1 5} \mathrm{K}$ and $\boldsymbol{B}=\mathbf{1 5 8} \mathrm{G}$. a An illustration of the coherent control measurement scheme. EOM frequency is tuned to the energy difference between the $m_{\mathrm{s}}=0$ and $m_{\mathrm{s}}=-1$ and a $5 \mathrm{~ms}$ selective excitation polarizes the defect into $m_{\mathrm{s}}=+1$ sublevel. At this point, any microwave pulse sequence can be used to coherently control the population in the $m_{\mathrm{s}}=0,+1$ manifold. The population in the $m_{\mathrm{s}}=0$ is probed by a short $50 \mu$ s laser pulse and the PLE is measured by integrating the transient photoluminescence excitation for $155 \mu \mathrm{s}$. b Resonantly driven Rabi oscillations of the ground state spin measured with a $63(1) \%$ contrast and a decay time of 4.76 (7) $\mu$ s. c $5 \mathrm{MHz}$ detuned Ramsey interferometry measurement. A fit to the data reveals a $T_{2}{ }^{*}=307(17) \mathrm{ns}$. $\mathbf{d}$ A Hahn echo measurement reveals a $T_{2}=81(2) \mu \mathrm{s}$ with characteristic oscillations of ${ }^{13} \mathrm{C}(87.5(3) \mathrm{kHz})$ and ${ }^{29} \mathrm{Si}(68.0(1) \mathrm{kHz})$ Larmor precession frequencies at the field. e High contrast Rabi oscillations measured by reducing the probe time to $1 \mu \mathrm{s}$. The total signal is reduced but the contrast is increased to $79(2) \%$. All fits are shown in red and the error bars are the one sigma of the data.

Finally, we time resolve the polarization dynamics of $\mathrm{Cr}^{4+}$ to investigate defect initialization. We sweep the resonant excitation time using the same parameters in Fig. $3 b-d$ and measure the populations of the $m_{s}=0$ and the $m_{s}=+1$ spin sublevels (Fig. $4 \mathrm{c}$ ). When the resonant excitation time of the $m_{s}=0$ is less than the optical lifetime of $155 \mu \mathrm{s}$, the population in the excited state transferred from the $m_{\mathrm{s}}=0$ increases. Past that point in time, the $m_{\mathrm{s}}=0$ population depletes as the sub-ensemble polarizes into the $m_{\mathrm{s}}=+1$. Using a $\pi$-pulse to coherently swap the populations between the $m_{\mathrm{s}}=0$ and the $m_{\mathrm{s}}=+1$ states, we measure the polarized population within the $m_{\mathrm{s}}=+1$ sublevel which increases monotonically with the laser excitation time. The difference of the two traces is the optical contrast shown in Fig. 4d. An exponential fit to the data yields a rise time of $1.27(3) \mathrm{ms}$, corresponding to polarization within $\sim 10$ optical lifetimes with maximum contrast at $64(2) \%$. In order to achieve the reported $63 \%$ contrast for a $50 \mu \mathrm{s}$ probe time, we polarized the defect for $5 \mathrm{~ms}$. Reducing the optical lifetime through Purcell enhancement in an optical cavity would significantly decrease this initialization time.

\section{DISCUSSION}

In summary, we have demonstrated successful creation and activation of $\mathrm{Cr}^{4+}$ ions with exceptional optical and spin properties in commercial semi-insulating $\mathrm{SiC}$ substrates. This method could be used to introduce other transition metal ions such as $\mathrm{V}^{18,19}$ and $\mathrm{Mo}^{20}$ into $\mathrm{SiC}$ and GaN. ${ }^{10}$ The reported defect creation technique can also be extended to three-dimensional localization of single transition metal ions in $\mathrm{SiC}$ for device integration through nanoimplantation. ${ }^{30,31}$ For example, a single $\mathrm{Cr}^{4+}$ can be detected by placing it into a photonic cavity to reduce the excited state lifetime by Purcell enhancement. ${ }^{15}$ Within such a device, the already large fraction of indistinguishable photons in the neartelecom ZPL would be further enhanced. ${ }^{4} \mathrm{~A}$ dense ensemble of
$\mathrm{Cr}^{4+}$ ions within a cavity may achieve population inversion through the second-excited state to realize a laser. ${ }^{32}$ This ensemble can also be used to realize an optical memory where the optical qubit is stored within the collective ground state spin wave, limited by the $T_{2} \cdot{ }^{33}$ All these possible photonics applications readily come with integrated spins that can be initialized and read out with a high fidelity of at least $79 \%$. Furthermore, the measured ensemble spin coherence time $T_{2}$ of $81 \mu \mathrm{s}$ is spin-spin limited, showing that the potential of an isolated $\mathrm{Cr}^{4+}$ in $\mathrm{SiC}$ as spin qubits. $^{28}$

Finally, our results can be generalized to a broader set of transition metal ions in $d^{2}$ electronic configuration in a host that provides a strong tetrahedral ligand field. The $31 \mathrm{MHz}$ ensemble optical linewidth and the $79 \%$ readout fidelity combined with $T_{1}$ time greater than $1 \mathrm{~s}$ makes these class of transition metal electronic structures a promising set of transportable optically active spin qubits.

\section{METHODS}

Sample preparation

In Fig. 1c the substrates were high purity semi-insulating, on-axis $4 \mathrm{H}-\mathrm{SiC}$ sourced by Norstel $\mathrm{AB}$. The $\mathrm{SiC}$ samples were co-implanted with ${ }^{52} \mathrm{Cr}$ at $190 \mathrm{keV}$ energy with a dose of $5 \times 10^{11} \mathrm{~cm}^{-2}$, and ${ }^{12} \mathrm{C}$ at $100 \mathrm{keV}$ energy with a dose of $5 \times 10^{11} \mathrm{~cm}^{-2}$ by CuttingEdge lons, LLC. Both ions were hot implanted with a $7^{\circ}$ tilt at a temperature of $600^{\circ} \mathrm{C}$. Chromium atoms are calculated to have an average depth of $110 \mathrm{~nm}$ and a $30 \mathrm{~nm}$ straggle using the program Stopping Range of lons in Matter (SRIM). All samples were subsequently cleaned with organic solvents. A photoresist layer (AZ1518) is spun $\sim 2 \mu \mathrm{m}$ thick, baked at $95^{\circ} \mathrm{C}$ for 1 minute then $350^{\circ} \mathrm{C}$ for $30 \mathrm{~min}$, as a protective surface coating by forming a graphite cap during the high temperature annealing. The samples are annealed in a tube furnace with high purity $\mathrm{Ar}$ at $800-1400^{\circ} \mathrm{C}$ for 15 min with ramp rates of $100^{\circ} \mathrm{C}$ per hour. The annealing of the samples at $1500-1800^{\circ} \mathrm{C}$ was performed by Fraunhofer IISB in an Ar environment for 15 min with ramp rates of $900^{\circ} \mathrm{C}$ 
a

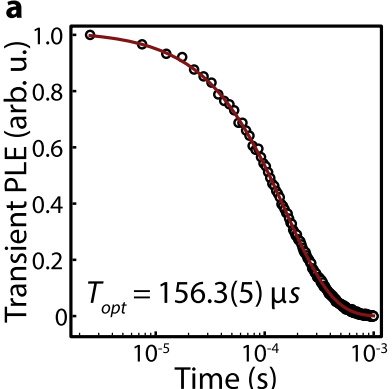

C

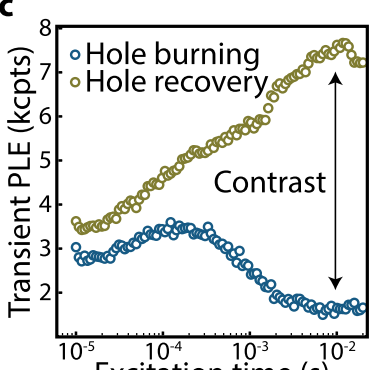

b

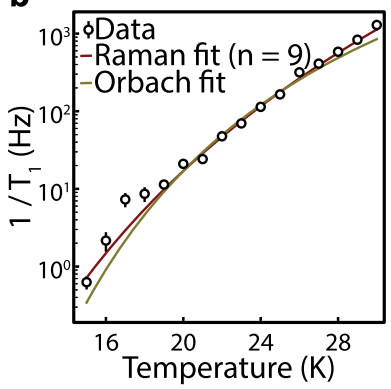

d

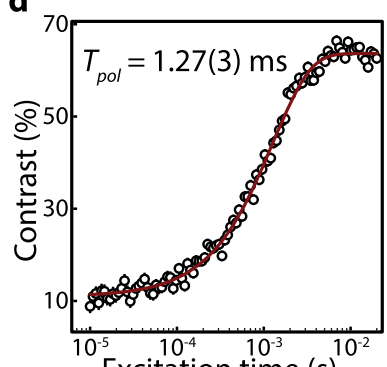

Excitation time $(\mathrm{s})^{10^{-4}}$

Fig. 4 Governing rates of $\mathbf{4 H}-\mathrm{SiC}_{\mathbf{C}} \mathbf{C r}_{\mathrm{A}}$. a Normalized photoluminescence excitation decay time at $T=30 \mathrm{~K}$ yields a $T_{\text {opt }}=156.3(5) \mu \mathrm{s}$. b Ground state spin $T_{1}$. After polarization, the contrast between a $\pi$ pulse and a $2 \pi$-pulse is measured at various temperatures. The resulting time-dependences are fit to an exponential decay, where the displayed error bars are the standard errors of the fits. The extracted $T_{1}$ times are fit to both an exponential (Orbach) and a power (Raman) model. The exponential fit (green line) reveals an $E=20(1) \mathrm{meV}$ with a reduced $x^{2}=4.18$. The 9 th power Raman fit (red line) reveals a constant temperature miscalibration of $\Delta T=3.2(5) \mathrm{K}$ with a reduced $X^{2}=1.91$. c Polarization dynamics at $T=15 \mathrm{~K}$ and $B=158 \mathrm{G} . m_{\mathrm{s}}=$ 0 state is probed as a function of polarization pulse length. In the absence of a $\pi$-pulse (blue trace) the depletion of the $m_{\mathrm{s}}=0$ state is measured. When a $\pi$-pulse is applied, the accumulation of the polarized population in the $m_{\mathrm{s}}=+1$ is measured by coherently transferring the population to $m_{\mathrm{s}}=0$ (green trace). $\mathbf{d}$ The contrast is the difference between the two traces in c. An exponential fit to the contrast shown in red yields a polarization time of $1.27(3)$ ms with a maximum contrast of $64(2) \%$ for a $50 \mu$ s probe time. The error bars in plots (a), (c), and (d) are smaller than the mark size and are not shown.

per hour. The graphite cap was removed by heating the sample to $750{ }^{\circ} \mathrm{C}$ for $30 \mathrm{~min}$ in air.

The box profile implanted sample in Figs. 1b, c, 2-4 used an epitaxial, undoped (intrinsic), $20 \mu \mathrm{m}$ thick $4 \mathrm{H}-\mathrm{SiC}$ layer that was grown on a $4^{\circ}$ offaxis semi-insulating $\mathrm{SiC}$ substrate sourced by Norstel $\mathrm{AB}$. The $4 \mathrm{H}-\mathrm{SiC}$ sample was implanted with ${ }^{52} \mathrm{Cr}$ ions in four steps with energies of $10,5,2$, $1 \mathrm{MeV}$ and doses of $(3,3,2,1.5) \times 10^{12} \mathrm{~cm}^{-2}$, respectively, at a sample temperature of approximately $690^{\circ} \mathrm{C}$ at Sandia National Laboratories. After ${ }^{52} \mathrm{Cr}$ implantation the sample was cleaned, photoresist capped, and annealed at $1800^{\circ} \mathrm{C}$ for 15 minutes using the same procedures detailed above.

\section{Optical and spin characterization experiments}

A closed cycle cryostat (Montana Instruments Nanoscale Workstation), with microwave and optical access is used to cool the sample. A NIR 50x objective (Olympus) is mounted to a heated and isolated shroud within the cryostat. Three in-cryo piezo stages (Attocube), each with a $4 \mathrm{~mm}$ range enabled switching between samples and locating microwave striplines.

A Thorlabs TO can $730 \mathrm{~nm}$ laser is used for off-resonant excitation. Resonant excitation is achieved by a fiber-coupled, external-cavity diode laser with a tuning range of $1035-1075 \mathrm{~nm}$ and a linewidth $<200 \mathrm{kHz}$ integrated over $50 \mathrm{~ms}$ with $18 \mathrm{~mW}$ maximum output power (Newfocus Velocity 6700). An electro-optical modulator (iXBlue) with a $12 \mathrm{GHz}$ bandwidth that generates optical side-bands is used for the experiments that needed two colors. A fiber coupled acousto-optical modulator
(Aaoptoelectronic) is used to rapidly turn the laser on and off, a second one is added for the $T_{1}$ data in Fig. $4 \mathrm{~b}$ to improve the extinction ratio over long wait times.

A vector signal generator (Stanford Research Instruments SG396) with a $30 \mathrm{~W}$ 0.6-2.7 GHz amplifier (Minicircuits) is used for microwave excitation. It is fed to a shorted coplanar wave guide patterned on a custom-made printed circuit board situated behind the sample within the cryostat.

For spectrally resolved measurements a liquid nitrogen cooled InGaAs NIR camera with a spectrometer (Princeton Instruments) is used. For resonant measurements, a superconducting nanowire single photon detector that is optimized for $1.0-1.2 \mu \mathrm{m}$ regime coupled to a $25 \mu \mathrm{m}$ multimode fiber (Quantum-Opus LLC) is used. The average dark count resulting from thermal photons slowly varying between 6 and $9 \mathrm{k}$ counts/s were measured before an experiment and subtracted from the data. The detection signal is gated using a microwave switch (Minicurcuits) and counted using a data acquisition system (National Instruments).

An arbitrary waveform generator (Swabian Instruments) is used to synchronize timings of the laser excitation, probe generation, microwave excitation with IQ control, collection, and counter gating.

\section{DATA AVAILABILITY}

All the data that support the findings of this study are available from the corresponding author upon reasonable request.

Received: 18 September 2019; Accepted: 2 January 2020; Published online: 29 January 2020

\section{REFERENCES}

1. Awschalom, D. D., Hanson, R., Wrachtrup, J. \& Zhou, B. B. Quantum technologies with optically interfaced solid-state spins. Nat. Photonics 12, 516-527 (2018).

2. Atatüre, M., Englund, D., Vamivakas, N., Lee, S.-Y. \& Wrachtrup, J. Material platforms for spin-based photonic quantum technologies. Nat. Rev. Mater. 3, 38-51 (2018).

3. Heremans, F. J., Yale, C. G. \& Awschalom, D. D. Control of spin defects in widebandgap semiconductors for quantum technologies. Proc. IEEE 104, 2009-2023 (2016).

4. Hensen, B. et al. Loophole-free Bell inequality violation using electron spins separated by 1.3 kilometres. Nature 526, 682-686 (2015).

5. Doherty, M. W. et al. The nitrogen-vacancy colour centre in diamond. Phys. Rep. 528, 1-45 (2013).

6. Bradac, C., Gao, W., Forneris, J., Trusheim, M. E., \& Aharonovich, I. Quantum nanophotonics with group IV defects in diamond. Nat. Commun. 10, 5625 (2019). https://doi.org/10.1038/s41467-019-13332-w.

7. Koehl, W. F., Buckley, B. B., Heremans, F. J., Calusine, G. \& Awschalom, D. D. Room temperature coherent control of defect spin qubits in silicon carbide. Nature 479, 84-87 (2011)

8. Christle, D. J. et al. Isolated spin qubits in SiC with a high-fidelity infrared spin-tophoton interface. Phys. Rev. X 7, 021046 (2017).

9. Nagy, R. et al. High-fidelity spin and optical control of single silicon-vacancy centres in silicon carbide. Nat. Commun. 10, 1954 (2019).

10. Koehl, W. F. et al. Resonant optical spectroscopy and coherent control of $\mathrm{Cr} 4+$ spin ensembles in SiC and GaN. Phys. Rev. B 95, 035207 (2017).

11. Kunkel, N. \& Goldner, P. Recent advances in rare earth doped inorganic crystalline materials for quantum information processing. Z. Anorg. Allg. Chem. 644, 66-76 (2018).

12. Dieke, G. H., Crosswhite, H. M. \& Dunn, B. Emission spectra of the doubly and triply ionized rare earths. J. Opt. Soc. Am. 51, 820 (1961).

13. Abragam, A. \& Bleaney, B. (Brebis). in Electron Paramagnetic Resonance of Transition lons 277-345 (Oxford University Press, 2012).

14. Zhong, T. et al. Nanophotonic rare-earth quantum memory with optically con trolled retrieval. Science 357, 1392-1395 (2017).

15. Dibos, A. M., Raha, M., Phenicie, C. M. \& Thompson, J. D. Atomic source of single photons in the telecom band. Phys. Rev. Lett. 120, 243601 (2018).

16. Raha, M. et al. Optical quantum nondemolition measurement of a solid-state spin without a cycling transition. Preprint at arXiv:1907.09992 (2019).

17. Figgis, B. N. \& Hitchman, M. A. Ligand Field Theory and Its Applications (Special Topics in Inorganic Chemistry) (Wiley, 1999).

18. Spindlberger, L. et al. Optical properties of vanadium in $4 \mathrm{H}$ silicon carbide for quantum technology. Phys. Rev. Appl. 12, 014015 (2019).

19. Wolfowicz, G. et al. Vanadium spin qubits as telecom quantum emitters in silicon carbide. Preprint at arXiv:1908.09817 (2019). 
20. Bosma, T. et al. Identification and tunable optical coherent control of transitionmetal spins in silicon carbide. npj Quantum Inf. 4, 48 (2018).

21. Pingault, B. et al. Coherent control of the silicon-vacancy spin in diamond. Nat. Commun. 8, 15579 (2017).

22. Son, N. T. et al. Photoluminescence and Zeeman effect in chromium-doped $4 \mathrm{H}$ and 6H SiC. J. Appl. Phys. 86, 4348-4353 (1999).

23. Ohno, K. et al. Three-dimensional localization of spins in diamond using $12 \mathrm{C}$ implantation. Appl. Phys. Lett. 105, 052406 (2014).

24. Falk, A. L. et al. Polytype control of spin qubits in silicon carbide. Nat. Commun. 4, 1819 (2013).

25. Cardona, M. \& Thewalt, M. L. W. Isotope effects on the optical spectra of semiconductors. Rev. Modern Phys. 77, 1173 (2005).

26. Imbusch, G. F., Yen, W. M., Schawlow, A. L., Devlin, G. E. \& Remeika, J. P. Isotope shift in the $R$ lines of chromium in ruby and MgO. Phys. Rev. 136, A481-A486 (1964).

27. Anderson, C. P. et al. Electrical and optical control of single spins integrated in scalable semiconductor devices. Science 366, 1225-1230 (2019).

28. Christle, D. J. et al. Isolated electron spins in silicon carbide with millisecond coherence times. Nat. Mater. 14, 160-163 (2015).

29. Castner, T. G. Jr. Raman spin-lattice relaxation of shallow donors in silicon. Phys. Rev. 491, 58-75 (1963).

30. Jamieson, D. N. et al. Deterministic doping. Mater. Sci. Semicond. Process. 62, 23-30 (2017).

31. Pacheco, J. L. et al. Ion implantation for deterministic single atom devices. Rev. Sci. Instrum. 88, 123301 (2017).

32. Kück, S. Laser-related spectroscopy of ion-doped crystals for tunable solid-state lasers. Appl. Phys. B Lasers Opt. 72, 515-562 (2001).

33. Afzelius, M., Simon, C., de Riedmatten, H. \& Gisin, N. Multimode quantum memory based on atomic frequency combs. Phys. Rev. A 79, 052329 (2009).

34. Ferro, G. \& Chaussende, D. A new model for in situ nitrogen incorporation into 4H-SiC during epitaxy. Sci. Rep. 7, 43069 (2017).

\section{ACKNOWLEDGEMENTS}

The authors thank S. Bayliss, M. Fataftah, D.W. Laorenza, M.K. Wojnar, T. Fidler, and A. Bauer for useful discussion. This project was supported by the US Department of Energy, Office of Science, Basic Energy Sciences, Materials Sciences and Engineering Division. This work was performed, in part, at the Center for Integrated Nanotechnologies, an Office of Science User Facility operated for the U.S. Department of Energy (DOE) Office of Science. Sandia National Laboratories is a multimission laboratory managed and operated by National Technology \& Engineering Solutions of Sandia, LLC, a wholly owned subsidiary of Honeywell International, Inc., for the U.S. DOE's National Nuclear Security Administration under contract DE-NA-0003525. The views expressed in the article do not necessarily represent the views of the U.S. DOE or the United States Government.

\section{AUTHOR CONTRIBUTIONS}

B.D. performed the experiments and analyzed the data. S.J.W. and C.P.A. annealed and cleaned the samples and E.S.B. implanted the box profile implanted sample. M.W. deposited an IR coating to enhance the signal that was used for the initial exploration. G.W. provided experimental guidance. D.D.A. and F.J.H. advised and coordinated all efforts. All authors contributed to the manuscript.

\section{COMPETING INTERESTS}

The authors declare no competing interests.

\section{ADDITIONAL INFORMATION}

Supplementary information is available for this paper at https://doi.org/10.1038/ s41534-020-0247-7.

Correspondence and requests for materials should be addressed to D.D.A.

Reprints and permission information is available at http://www.nature.com/ reprints

Publisher's note Springer Nature remains neutral with regard to jurisdictional claims in published maps and institutional affiliations.

(i) Open Access This article is licensed under a Creative Commons Attribution 4.0 International License, which permits use, sharing, adaptation, distribution and reproduction in any medium or format, as long as you give appropriate credit to the original author(s) and the source, provide a link to the Creative Commons license, and indicate if changes were made. The images or other third party material in this article are included in the article's Creative Commons license, unless indicated otherwise in a credit line to the material. If material is not included in the article's Creative Commons license and your intended use is not permitted by statutory regulation or exceeds the permitted use, you will need to obtain permission directly from the copyright holder. To view a copy of this license, visit http://creativecommons. org/licenses/by/4.0/.

(c) The Author(s) 2020 